\title{
Inhaled budesonide for acute asthma - is it all a question of time and space?
}

\author{
Emanuel Sarinho*
}

I synthesis of products with maximum topical effects and minimum systemic potential. This combination has only become possible with the development by industry of liposoluble agents with a high level of affinity for receptors and are rapidly deactivated by the liver after systemic absorption with a resulting reduction in the risk of side effects.

Bronchospasm, edema and hypersecretion coexist in acute asthma and, in this context, exacerbation of the pulmonary inflammatory process. According to a number of different consensus documents, including the Third Brazilian Consensus on Asthma Management, in acute crises systemic corticoids allow greater functional recuperation, reduce relapses and should be started soon early during the asthma crisis. ${ }^{1}$

A systematic review of the Cochrane Library on acute asthma treatment, found, through 12 studies involving 863 patients that the early use of systemic corticoids resulted in a significant reduction in hospitalization rates among more severe cases. Response levels to oral corticoids were particularly effective among children. ${ }^{2}$

The most important role of corticoids in asthma appears to be deactivation of multiple inflammatory genes, normally activated within these patients' airways. Overall, the significant biological actions performed by this group of medications in acute asthma are: the suppression of the synthesis of Th2 cytokines such as IL-5 thus reducing eosinophilic infiltration into the airways, the reduction of plasma exudation with consequent mucus secretion, and the reduction of inflammatory mediators such as leukotrienes and prostaglandins. ${ }^{3}$

Inhaled steroids have greater anti-asthmatic potential and anti-inflammatory power than do oral steroids and can offer rapid results during a crises since they act directly on the lungs. This being the case, the following research question is posed: "Could inhaled steroids be indicated for acute asthma?".4

Following this path, and choosing the question: "Is there a difference in efficacy between oral prednisone and inhaled budesonide for acute asthmatic crisis treatment?" Milani et al. planned a clinical trial entitled "Nebulized

\footnotetext{
* PhD. Associate professor. Coordinator of the Research Center of Allergy and Immunology in Pediatrics, Hospital das Clínicas, Universidade Federal de Pernambuco, Recife, PE, Brazil.
}

budesonide to treat acute asthma in children" which is of great relevance for daily practice since it incorporates the recommendation of steroids for moderate acute episodes when there is an incomplete response or a recurrence of symptoms after inhaled b2-agonist has been used. 5

The study design employing double-placebo made three comparison groups possible: the conduct to be tested (inhaled budesonide), standard conduct (oral corticoids) and no steroid treatment (double-placebo group). Ethically, the real need for this last group could be questioned, since oral prednisone is well established for acute asthma, but the most important point is that severe asthmatics were excluded. Overall, methodologically speaking, the presence of the doubleplacebo makes the research more elegant by indirectly reviewing whether the standard treatment for acute asthma, oral corticoids, was effective in this sample.

The choice of a period of wheezing longer than six hours' duration, which does not appear to follow normal distribution, as one of the inclusion criteria allowed for a reliable classification of asthma. An earlier cut-off may have defined crises in evolution. On the other hand it was, possibly, important to have excluded patients wheezing for more than 24 hours since it is known that after this point there is less chance of a satisfactory response to the treatment, in particular response to a single dose of steroids which could have acted as a confounding factor, but it appears that this situation occurred in few cases and probably did not compromise the study.

It is important to point out that the operating procedure of this research was further refined by including patients after the use of beta2-agonist inhalation and by the excluding the most severe cases. The use of a clinical severity score and of saturation measurement with results expressed graphically also confers significant scientific validity on the work.

Despite the final outcome of the study demonstrating that improvements in the acute asthma clinical score were similar for all three groups (oral prednisone vs. inhaled budesonide vs. placebo), doubt remains as to whether this represents a negative result or an inconclusive one since there is a chance that sample size was underestimated. One fact of general interest is that one of the fifteen moderate asthma patients in the placebo group deteriorated to the extent of requiring hospitalization and was excluded from analysis. This fact, taken together with delays of 
more than 24 hours for significant saturation improvement among patients in this group, may indicate the superiority of the oral prednisone oral and inhaled budesonide groups, which is in agreement with biological logic.

Seen from a different angle, it can be inferred that a single dose of budesonide is effective to a similar extent as conventional treatment. This may have been the opinion of the authors when they state that "single-dose inhaled budesonide did not reveal any superiority over the conventional treatment of moderate asthma crises with oral prednisone". Budesonide is a non-halogenated corticosteroid which can be prepared in solution for nebulization in which case it appears to have greater efficacy during crises due to better binding with the intracellular lipophilic receptor than sprays. An immediate antiinflammatory effect is suggested by reduced eosinophil levels in sputum after a single $2.4 \mathrm{mg}$ dose. In children it has further been demonstrated that nebulization with 2 $\mathrm{mg}$ of the drug reduces nitric oxide excretion and reduces asthma symptoms as assessed between 6 and 12 hours later. ${ }^{6}$ Nevertheless, systematic reviews of scientific literature claim that there is not sufficient scientific evidence to support the use of inhaled steroids as effective for urgent treatment of acute asthma crises ${ }^{7}$ but even so, studies are appearing, including of children, that demonstrate inhaled medication is better than a placebo. ${ }^{8}$

Oxygen saturation measurement, considered the fifth vital sign, is extremely sensitive for acute asthma assessment, indicating requirements for medical care, severity of the crises and risk of death. Analyzing the study through this lens, the fact that the oral prednisone group exhibited oxygen saturation improvement, in comparison with the baseline level, that was already significant after just two hours of treatment may indicate therapeutic superiority. It is possible that the better response to oral prednisone was the result of an elevated dose, but this does not seem to be the case since only $1 \mathrm{mg} / \mathrm{kg}$ of this corticoid was used in contrast with $2 \mathrm{mg}$ of budesonide. A classic study by Toogood et al. in 1989 of patients with asthma found that budesonide had an anti-asthmatic potential 58 times greater than prednisone with systemic potential just nine times smaller. ${ }^{9}$ This being the case, it would be expected that the inhaled corticoid should work better as it is deposited rapidly at the point of action. ${ }^{9}$

Is oral prednisone, therefore, a magical drug? After all, having passed through the entire process of intestinal absorption, passing the liver, and being distributed through tissues until reaching the lungs, how can the improved oxygen saturation in less than two hours be explained when budesonide exhibits a delayed effect? There are three explanatory hypotheseses:

- it could be that pulmonary availability of the drug inhaled during a crises is lower than that supplied by the oral route;

- it is possible that inhaled budesonide could present an immediate response in acute asthma because of small airway compromise and excess of trapped mucus;
- it is even possible that, in an acute asthma crisis, an effective systemic action is required of the corticoid for quick clinical improvement.

Perhaps systemic corticoids subdue the immediate inflammatory reaction of an acute asthma attack while inhaled steroids are incapable of achieving this objective. Beneficial biochemical effects appear in an acute asthma case after oral corticoid is administered, such as reduced serum interleukin 5 levels, interleukin-2(CD25) receptor levels and eosinophil cationic protein levels. ${ }^{3}$

Studies of inhaled corticoid during asthma exacerbation have returned conflicting results as a result of varying doses and medications and also of differing study designs and outcomes, which impedes adequate comparison. An interesting approach suggests that budesonide could be an effective adjunct to prednisone for infants with acute asthma, 10 but as it implies increased costs the true benefit must be better analyzed.

Finally, while pharmaceutical acute asthma management has innumerable controversial issues, according to currently available scientific evidence, oral prednisone is effective, low cost and easy to administer. This, however, is neither set in stone nor fixed dogma since science evolves through both time and space. ${ }^{1,11}$

\section{References}

1. III Consenso Brasileiro no Manejo da Asma. J. Pneumol. 2002;28 Supl 1:1-28.

2. Rowe BH, Spooner C, Ducharme FM, Bretzlaff JA, Bota GW. Early emergency department treatment of acute asthma with systemic corticosteroids (Cochrane Review). In: The Cochrane Library, Issue 1, 2004. Chichester, UK: John Wiley \& Sons Ltd.; 2004.

3. Sahid El-Radhi A, Hogg CL, Bungre JK, Bush A, Corrigan CJ. Effect of oral glucocorticoid treatment on serum inflammatory markers in acute asthma. Arch Dis Child. 2000;83:158-62.

4. De Blic J, Delacourt C, Le Bourgeois M, Mahut B, Ostinelli J, Caswell C, et al. Efficacy of nebulized budesonide in treatment of severe infantile asthma: a double-blind study. J Allergy Clin Immunol. 1996;98:14-20.

5. Milani GKM, Rosário Filho NA, Riedi CA, Figueiredo BC. Budesonida inalatória em crianças com asma aguda. J Pediatr (Rio J). 2004;80:106-12.

6. Tsai Y-G, Lee M-Y, Yang KD, Chu D-M, Yuh SY, Hung C-H. A single dose of nebulized budesonide decreases exhaled nitric oxide in children with acute asthma. J Pediatr. 2001;139:433-7.

7. Edmonds ML, Camargo CA Jr, Pollack CV Jr, Rowe BH. Early use of inhaled corticosteroids in the emergency department treatment of acute asthma (Cochrane Review). In: The Cochrane Library, Issue 1, 2004. Chichester, UK: John Wiley \& Sons Ltd.; 2004.

8. Volovitz B, Nussinovitch M, Finkelstein Y, Harel L, Varsano I. Effectiveness of inhaled corticosteroids in controlling acute asthma exacerbations in children at home. Clin Pediatr (Phil). 2001;40:79-86.

9. Toogood JH, Baskerville J, Jennings B, Lefcoe NM, Johansson SA. Bioequivalent doses of budesonide and prednisone in moderate and severe asthma. J Allergy Clin Immunol. 1989;84 Pt 1:688-700.

10. Sung L, Osmond MH, Klassen TP. Randomized controlled trial of inhaled budesonide as an adjunct to oral prednisone in acute asthma. Acad Emerg Med. 1998;5:209-13.

11. Amantéa SL, Sanchez I, Piva JP, Garcia PCR. Controvérsias no manejo farmacológico da asma aguda infantil. J Pediatr (Rio J). 2002;78 Supl 2:151-60.

Corresponding author:

Emanuel Sarinho

Av. Parnamirin, 278/12

CEP 52060-000 - Recife, PE, Brazil

E-mail: emanuelsarinho@uol.com.br 